\title{
The allelic distribution of a single nucleotide polymorphism in the PDCD5 gene locus of Japanese non-small cell lung cancer patients
}

\author{
KEI NANBA ${ }^{1}$, SHINICHI TOYOOKA ${ }^{1}$, JUNICHI SOH ${ }^{1}$, KAZUNORI TSUKUDA ${ }^{1}$, HIROMASA YAMAMOTO ${ }^{1}$, \\ AKIKO SAKAI $^{2}$, MAMORU OUCHIDA ${ }^{2}$, NARUYUKI KOBAYASHI ${ }^{1}$, KEITARO MATSUO $^{5}$, NORIO KOIDE ${ }^{3}$, \\ KATSUYASU KUSAKA ${ }^{4}$, KENJI SHIMIZU ${ }^{2}$ and HIROSHI DATE ${ }^{6}$
}

\begin{abstract}
Departments of ${ }^{1}$ Cancer and Thoracic Surgery, ${ }^{2}$ Molecular Genetics, and ${ }^{3}$ Laboratory Medicine, Graduate School of Medicine, Dentistry and Pharmaceutical Sciences, Okayama University, Okayama; ${ }^{4}$ Department of Internal Medicine, Kusaka Hospital, Bizen, Okayama; ${ }^{5}$ Division of Epidemiology and Prevention, Aichi Cancer Center Research Institute, Nagoya;

${ }^{6}$ Department of Thoracic Surgery, Graduate School of Medicine, Kyoto University, Kyoto, Japan
\end{abstract}

Received April 3, 2008; Accepted June 9, 2008

DOI: $10.3892 / \mathrm{mmr}_{0} 00000010$

\begin{abstract}
It has been reported that the rs1862214 single nucleotide polymorphism (SNP) in the programmed cell death 5 gene (PDCD5) is associated with smoking-related lung cancer risk and prognosis in a European population with a history of smoking. The aim of this study was to investigate the status and impact of SNPs in the PDCD5 locus of a Japanese population. We developed an assay based on realtime PCR with melting curve analysis for determining the rs 1862214 SNP, and examined this SNP in 165 lung cancer patients and 180 healthy volunteers. Of the 165 lung cancer patients (107 smokers), 25 (17), 72 (47) and 68 (43) had the CC, CG and GG genotypes of rs1862214, respectively. Of the 180 volunteers (117 smokers), 31 (24), 81 (52) and 68 (41) had the CC, CG and GG genotypes of rs1862214, respectively. No significant difference in allelic distribution was found between Japanese patients and healthy controls, even among smokers. Based on the published data, the distribution of this SNP appears to be significantly different in Japanese and European populations. No significant difference in prognosis according to the SNP was observed, either in patients with a history of smoking or in the total number of patients. This too differs from the results from a European population. In conclusion, we developed a convenient real-time PCR-based assay for the genotyping of rs1862214 in the PDCD5 locus. The distribution
\end{abstract}

Correspondence to: Dr Shinichi Toyooka, Department of Cancer and Thoracic Surgery, Graduate School of Medicine, Dentistry and Pharmaceutical Sciences, Okayama University, 2-5-1 Shikata-cho, Okayama 700-8558, Japan

E-mail: toyooka@md.okayama-u.ac.jp

Abbreviations: SNP, single nucleotide polymorphisms; NSCLC, non-small cell lung cancer

Key words: single nucleotide polymorphisms, PDCD5, Japanese, non-small cell lung cancer, cancer risk of the rs1862214 SNP in our Japanese population differs from its distribution in a European population, and is not related to the risk of cancer or to poor prognosis in lung cancer. This suggests the presence of an ethnicity-related difference in the role of PDCD5 in the pathogenesis of lung cancer.

\section{Introduction}

Lung cancer is the leading cause of cancer death in Japan and many other countries worldwide $(1,2)$. Survival among patients with non-small cell lung cancer remains unsatisfactory because many locally advanced or metastatic cases are unresectable. Even patients with the early stages of the disease who undergo complete resections often experience recurrence, resulting in an unfavorable prognosis. In short, until now the effectiveness of therapeutic strategies has been limited.

In addition to aiding in the development of therapeutic strategies, the prevention or early detection of cancer improves its rate of mortality. Several genetic polymorphisms are considered to be molecular markers that predict the risk, progression and prognosis of various cancers (3-7). Indeed, single nucleotide polymorphisms (SNPs) in several genes are reported to be associated with the risk of lung cancer (8-10), for example SNPs in the caspase-3 and vascular endothelial growth factor genes $(11,12)$.

Recently, Spinola et al reported that the rs1862214 SNP in the programmed cell death 5 gene (PDCD5) locus, which is known to be involved in apoptosis $(13,14)$, was associated with lung cancer risk and prognosis in a European population with a history of smoking (15). They performed a case-control study using German and Italian lung cancer patients along with controls enrolled at the same sites, and found that patients with genotypes GG or CG for PDCD5 showed an increased risk of lung cancer, a higher incidence of poor clinical stage disease and short-term survival compared to those with the common $\mathrm{CC}$ genotype. These findings indicate that PDCD5 may be a potential molecular marker, predicting the risk of lung cancer and prognosis. However, the impact of the PDCD5 SNP must be assessed before it is used to evaluate the risk and prognosis of lung cancer in other ethnicities, such as in a Japanese 
population, since the function of polymorphisms, including SNPs, is often affected by ethnicity $(16,17)$.

In the current study, we analyzed the allelic distribution of an SNP at rs1862214 in the PDCD5 locus of Japanese lung cancer patients and healthy controls, and studied its impact on the risk of lung cancer and on prognosis in a Japanese population.

\section{Materials and methods}

Patients and control groups. We analyzed 165 Japanese patients who were histologically diagnosed as having primary lung cancer and who had undergone surgery at Okayama University Hospital (Okayama, Japan) between 2000 and 2006. We recruited 180 healthy volunteers in Okayama prefecture, Japan. Institutional Review Board approval and informed consent from all lung cancer patients and controls were obtained. The characteristics of the 165 lung cancer patients and 180 controls are shown in Table I. There were no significant differences in sex, age or smoking status at the time of recruitment between the lung cancer patients and the controls.

DNA extraction and genotyping of rs1862214 in the PDCD5 locus. The genomic DNA of the 165 patients was isolated from freshly-frozen non-malignant peripheral lung tissue using SDS/proteinase K treatment, phenolchloroform extraction and ethanol precipitation. The genomic DNA of the 180 controls was extracted from peripheral lymphocytes.

To determine the genotype of rs1862214 in the PDCD5 locus with rapidity, a convenient genotyping assay using real-time PCR was developed. We designed the primers and hybridization probes for the rs 1862214 SNP in the PDCD5 locus to be used in real-time PCR, then detected the genotype of each sample by melting curve analysis. Two different fluorescent-labeled hybridization probes, which hybridize to an internal sequence of the amplified fragment, were added during PCR. One probe was labeled at the 3 '-end with fluorescein (anchor). The other was labeled at the 5'-end with a LightCycler-Red fluorophore (LC-Red 705) and, to avoid extension, modified at the 3'-end by phosphorylation (sensor).

Using the LightCycler 1.5 System (Roche Applied Science, Indianapolis, IN), real-time PCR was performed in capillaries with a reaction volume of $20 \mu 1$ containing $200 \mathrm{ng}$ of DNA, $0.5 \mu \mathrm{M}$ sense (5'-GCC AGA AGC AAG ACT GAT AC-3') and antisense (5'-CCT GAA AGG AAA CCC ACA TTT A-3') primers, $0.2 \mu \mathrm{M}$ anchor (5'-GGC CTC CTG GCC TCC TGT TTT CCT GAT CAC ACC AC-3') and sensor (5'-CAC TGC ACG AGC ATT TTC-3') hybridization probes, $1 \mathrm{X}$ reaction buffer (LightCycler FastStart DNA Master HybProbe, Roche Applied Science) and $2 \mathrm{mM} \mathrm{MgCl}_{2}$. The sensor hybridization probe was specific for the $\mathrm{G}$ allele. PCR conditions were as follows: one cycle at $95^{\circ} \mathrm{C}$ for $10 \mathrm{~min}, 45$ cycles at $95^{\circ} \mathrm{C}$ for $10 \mathrm{sec}, 61^{\circ} \mathrm{C}$ for $10 \mathrm{sec}$ and $72^{\circ} \mathrm{C}$ for $10 \mathrm{sec}$. After PCR, derivative melting curves $[(-\mathrm{dF} / \mathrm{dT})$ vs $\mathrm{T}]$ were generated by slowly heating the amplicon/probe heteroduplex and measuring the dramatic changes in fluorescence.

In order to genotype rs 1862214 , we also performed direct sequencing using 93 out of the 165 samples to confirm the reliability of the PCR-based convenient assay.
Table I. Characteristics of lung cancer patients and healthy controls.

\begin{tabular}{lccc}
\hline Characteristic & $\begin{array}{c}\text { Lung cancer } \\
\text { patients } \\
(\mathrm{n}=165)\end{array}$ & $\begin{array}{c}\text { Healthy } \\
\text { controls } \\
(\mathrm{n}=180)\end{array}$ & P-value \\
\hline Mean age (years $\pm \mathrm{SD})$ & $66.6 \pm 10.8$ & $68.1 \pm 11.4$ & 0.212 \\
Sex & & & \\
Male & 108 & 115 & 0.761 \\
Female & 57 & 65 & \\
Smoking status & & & \\
Non-smoker & 58 & 63 & 0.977 \\
Smoker & 107 & 117 & \\
\hline
\end{tabular}

$\mathrm{SD}$, standard deviation.

Statistical analysis. All statistical analyses were performed using SPSS 12.0 (SPSS Inc., Chicago, IL). The odds ratio (OR) and $95 \%$ confidence interval (CI) were adjusted for age, sex and smoking status using an unconditional logistic regression model. Accordance with Hardy-Weinberg equilibrium was determined for lung cancer patients and healthy controls using the $\chi^{2}$ test. P-values $<0.05$ were considered to be significant.

\section{Results}

Detection of rs 1862214 SNPs by real-time PCR with melting curve analysis. We investigated the distribution of the genotype of rs1862214 in the PDCD5 locus of Japanese lung cancer patients and healthy controls. Initially, 93 cases were tested by both PCR-based convenient assay and direct sequencing. The results of the assay were consistent with those of the direct sequencing analysis (Fig. 1). Consequently, we used the assay alone for the remaining cases, as it enabled us to rapidly determine the SNP.

Impact of the rs1862214 SNP in the PDCD5 locus on lung cancer risk. In 165 primary Japanese lung cancers, there were 25, 72 and 68 cases of CC, CG and GG genotypes, respectively (Table II). When limited to patients with a history of smoking, $17(15.9 \%)$ had a CC, $47(43.9 \%)$ had a CG and $43(40.2 \%)$ had a GG genotype. In 180 healthy volunteers, the incidence of the CC, CG and GG genotypes was 31, 81 and 68 , respectively. When limited to volunteers with a history of smoking, $24(20.5 \%)$ had a CC, $52(44.4 \%)$ had a CG and 41 $(35.0 \%)$ had a GG genotype. None of these numbers deviated from those expected according to Hardy-Weinberg equilibrium $(\mathrm{P}=0.870$ and 0.832 in total patients and total controls; $\mathrm{P}=0.878$ and 0.764 in smoking patients and smoking controls, respectively). There was no significant difference between the frequency of the $\mathrm{G}$ genotype in lung cancer patients and in healthy controls. In addition, in the Japanese population, there was no significant difference between the frequency of the $G$ genotype in lung cancer patients and in controls with a history of smoking. This is inconsistent with the frequencies previously 
A.

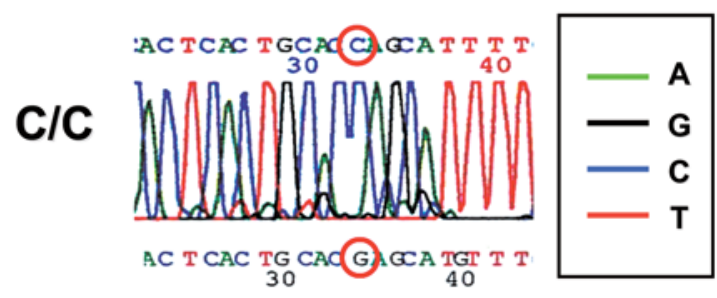

G/G

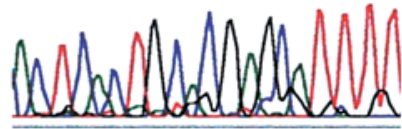

AC TCAC TGCACNGCA T T T T

C/G
B.

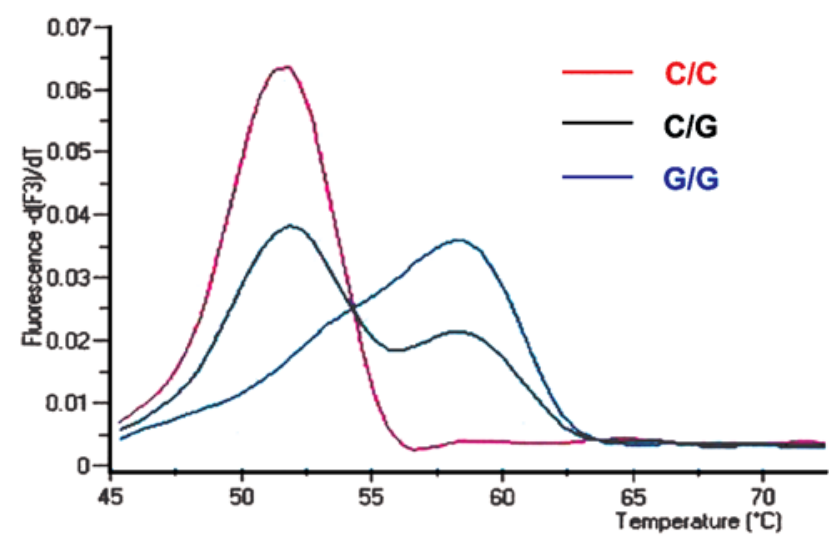

Melting peak temperature:

\section{G allele: $59.9^{\circ} \mathrm{C}$ \\ C allele: $52.6^{\circ} \mathrm{C}$}

Figure 1. Representative figures of direct sequencing and melting curve analysis for genotyping at rs1862214 in the PDCD5 locus. (A) Data from direct sequencing show three different genotypes of rs 1862214 in the PDCD5 locus. The top row shows the homozygous genotype of $\mathrm{C}$ (C/C), the middle row the homozygous genotype of $\mathrm{G}(\mathrm{G} / \mathrm{G})$, and the bottom row the heterozygous genotype of rs1862214 (C/G). (B) Melting curve analysis distinguishes three different genotypes of rs 1862214 . The homozygous genotype of $\mathrm{C}$ generates a melting curve with its peak at $52.6^{\circ} \mathrm{C}$. The homozygous genotype of $\mathrm{G}$ generates its peak at $59.9^{\circ} \mathrm{C}$. The heterozygous genotype generates double peaks at 52.6 and $59.9^{\circ} \mathrm{C}$.

Table II. Genotypes of rs 1862214 in the PDCD5 locus of Japanese lung cancer patients.

\begin{tabular}{|c|c|c|c|c|c|}
\hline & Controls ${ }^{a}$ & Patients $^{\mathrm{a}}$ & Odds ratio ${ }^{\mathrm{b}}$ & $95 \%$ CI & P-value \\
\hline \multicolumn{6}{|l|}{ Total cases } \\
\hline $\mathrm{C} / \mathrm{C}$ & 31 & 25 & 1.00 & & \\
\hline $\mathrm{C} / \mathrm{G}$ & 81 & 72 & 1.10 & $0.58-2.0$ & 0.797 \\
\hline $\mathrm{G} / \mathrm{G}$ & 68 & 68 & 1.20 & $0.66-2.3$ & 0.509 \\
\hline $\mathrm{C} / \mathrm{G}$ or $\mathrm{G} / \mathrm{G}$ & 149 & 140 & 1.20 & $0.65-2.1$ & 0.627 \\
\hline \multicolumn{6}{|l|}{ Smokers } \\
\hline $\mathrm{C} / \mathrm{C}$ & 24 & 17 & 1.00 & & \\
\hline $\mathrm{C} / \mathrm{G}$ & 52 & 47 & 1.30 & $0.62-2.7$ & 0.502 \\
\hline $\mathrm{G} / \mathrm{G}$ & 41 & 43 & 1.50 & $0.70-3.1$ & 0.310 \\
\hline $\mathrm{C} / \mathrm{G}$ or $\mathrm{G} / \mathrm{G}$ & 93 & 90 & 1.40 & $0.69-2.7$ & 0.366 \\
\hline \multicolumn{6}{|l|}{ Non-smokers } \\
\hline $\mathrm{C} / \mathrm{C}$ & 7 & 8 & 1.00 & & \\
\hline $\mathrm{C} / \mathrm{G}$ & 29 & 25 & 0.67 & $0.21-2.2$ & 0.497 \\
\hline $\mathrm{G} / \mathrm{G}$ & 27 & 25 & 0.76 & $0.24-2.4$ & 0.644 \\
\hline $\mathrm{C} / \mathrm{G}$ or $\mathrm{G} / \mathrm{G}$ & 56 & 50 & 0.71 & $0.24-2.1$ & 0.547 \\
\hline
\end{tabular}

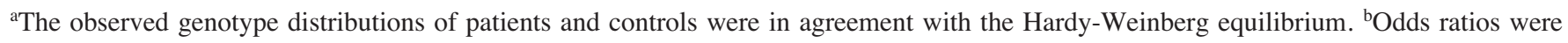
adjusted for age, sex and smoking status.

reported by Spinola et al (15) in a European population. Regarding ethnic differences in the genotype, in the Japanese population the $\mathrm{G}$ haplotype was dominant in both patients and in healthy volunteers. According to the report by Spinola et al, (15) this is rare in European patients and points to an ethnic difference between the Japanese and European populations.
Prognosis for lung cancer patients stratified by the rs 1862214 SNP in the PDCD5 locus. We examined the impact of the rs1862214 SNP in the PDCD5 locus on the prognosis for Japanese lung cancer patients. There was no significant difference between the prognosis for the $\mathrm{CC}$ and $\mathrm{G}$-contained variants (GG or CG). Since Spinola et al had found that the 
A.

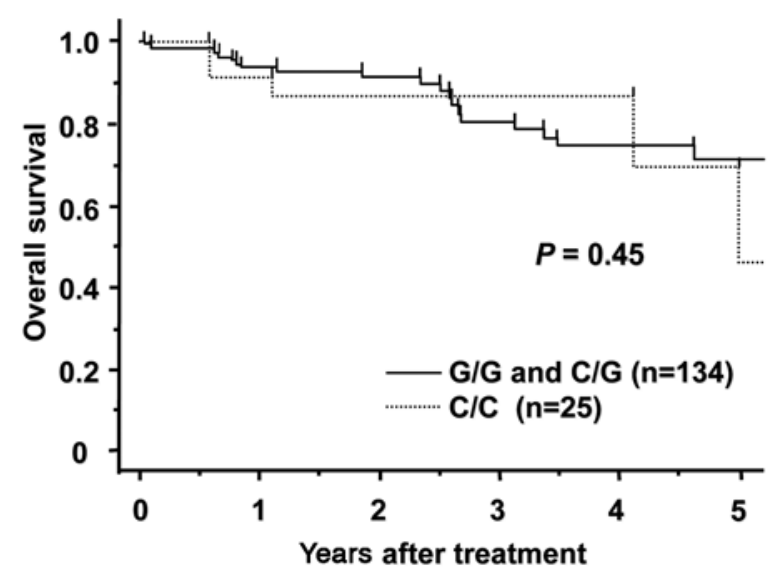

B.

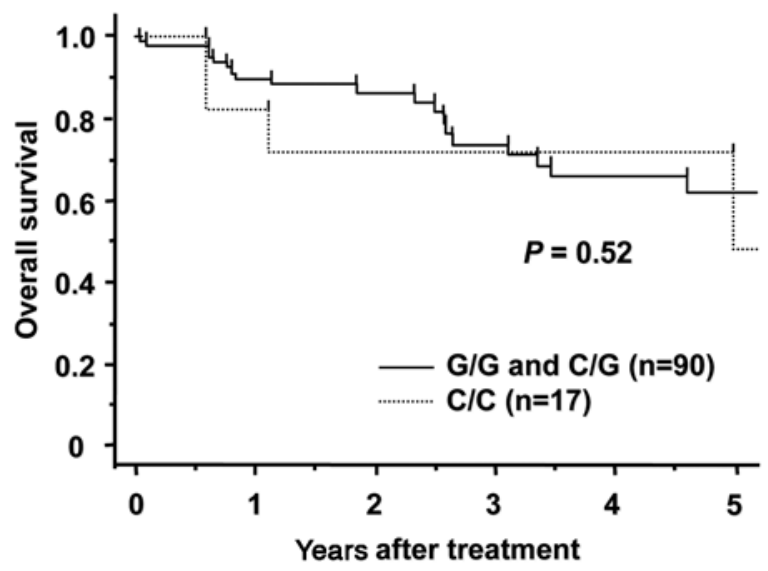

Figure 2. Kaplan-Meier curves showing overall survival according to the genotype of PDCD5. (A) Kaplan-Meier curves for all cases. (B) Kaplan-Meier curves for the smokers. No significant differences in overall survival were observed in the total number of patients (A) or in patients with a history of smoking (B) based on the genotype of rs 1862214 .

G-contained genotype had a significant prognostic impact on lung cancer patients with a history of smoking, we performed a similar analysis for our subgroups classified as having a history of smoking. However, even when restricted to smokers, no significant difference was observed between the prognosis for the two genotypes (Fig. 2).

\section{Discussion}

We developed a hybridization probe-based real-time PCR assay with melting curve analysis for genotyping rs 1862214 in the PDCD5 locus, the reliability of which was confirmed by direct sequencing. This convenient assay is less timeconsuming and more cost-effective than direct sequencing, and is useful for the analysis of SNPs. These benefits would be meaningful were SNP genotyping to be applied for screening in the clinical field. The genotype of other significant genes can be determined using this real-time PCR-based assay, and a large scale of samples can be dealt with.

The biological function of PDCD5 is the regulation of apoptosis. PDCD5 expression levels are significantly increased in cells undergoing apoptosis, and its protein translocation from the cytoplasm to the nucleus of apoptotic cells precedes the externalization of phosphatidylserine and the fragmentation of chromosome DNA (13). Spinola et al investigated whether the rs1862214 SNP had a functional role in PDCD5 mRNA expression in normal and tumor lung tissue (15). However, in healthy lung parenchyma, PDCD5 expression was similar in $\mathrm{CC}$ genotype and G-allele carriers. The functional difference of PDCD5 in both genotypes is therefore unclear.

In the present study, we investigated an SNP at rs1862214 in the PDCD5 locus of a Japanese population. As polymorphisms, including SNPs, generally display ethnic variations, our aim was to ascertain whether PDCD5 can be used as a molecular marker for the prediction of lung cancer risk and prognosis in a Japanese as well as in a European population (16). We found a different allelic distribution at rs 1862214 compared to that found by Spinola et al. In the Japanese population, haplotype $\mathrm{G}$ was more frequent than $\mathrm{C}$, while Spinola et al had reported that the frequency of the $\mathrm{G}$ allele was $22 \%$ in both a German and an Italian population. These results suggest that there is a significant difference between SNP distribution at rs1862214 in European and Japanese controls and cancer patients $(\mathrm{P}<0.0001)$. Additionally, there was no significant association between cancer risk and a specific genotype of rs 1862214 among the total population or among the smokers alone. Moreoever, there was no difference in prognosis according to genotype among the cases with a history of smoking, which again differed from the results taken from the European population with a history of smoking. Taken together, these findings suggest that the impact of an SNP in the PDCD5 locus on lung cancer may vary in Japanese and European populations, and that this SNP may not be useful for predicting the risk of lung cancer in Japanese patients.

One limitation of the present study is that the study population may not have been large enough to be conclusive. The degree of smoking can also be an important factor and should ideally be considered when estimating risk and survival. Thus, further study is necessary to elucidate the impact of the SNP at rs1862214 in a Japanese population on the risk of lung cancer and on clinical outcome.

\section{Acknowledgements}

This study was supported by a Grant-in Aid for Scientific Research from the Ministry of Education, Science, Sports, Culture and Technology of Japan.

\section{References}

1. Jemal A, Siegel R, Ward E, Ward E, Hao Y, Xu J, Murray T and Thun MJ: Cancer statistics, 2008. CA Cancer J Clin 58: 71-96, 2008. 2. Pirozynski M: 100 years of lung cancer. Respir Med 100: 2073-2084, 2006

3. Yamamoto H, Hanafusa H, Ouchida M, et al: Single nucleotide polymorphisms in the EXO1 gene and risk of colorectal cancer in a Japanese population. Carcinogenesis 26: 411-416, 2005.

4. Hirata H, Hinoda Y, Kawamoto K, et al: Mismatch repair gene $\mathrm{MSH} 3$ polymorphism is associated with the risk of sporadic prostate cancer. J Urol 79: 2020-2024, 2008.

5. Zhang J, Dou C, Song Y, Ji C, Gu S, Xie Y and Mao Y: Polymorphisms of tumor necrosis factor-alpha are associated with increased susceptibility to gastric cancer: a meta-analysis. J Hum Genet (Epub ahead of print). 
6. Lehnerdt GF, Franz P, Zaqoul A, et al: Overall and relapse-free survival in oropharyngeal and hypopharyngeal squamous cell carcinoma are associated with genotypes of T393C polymorphism of the GNAS1 gene. Clin Cancer Res 14: 1753-1758, 2008.

7. Musolino A, Naldi N, Bortesi B, et al: Immunoglobulin G fragment $\mathrm{C}$ receptor polymorphisms and clinical efficacy of trastuzumab-based therapy in patients with HER-2/neu-positive metastatic breast cancer. J Clin Oncol 26: 1789-1796, 2008.

8. Kanzaki H, Ouchida M, Hanafusa H, et al: The association between RAD18 Arg302Gln polymorphism and the risk of human non-small-cell lung cancer. J Cancer Res Clin Oncol 134: 211-217, 2008.

9. Kanzaki H, Hanafusa H, Yamamoto H, et al: Single nucleotide polymorphism at codon 133 of the RASSF1 gene is preferentially associated with human lung adenocarcinoma risk. Cancer Lett 238: 128-134, 2006.

10. Kanzaki H, Ouchida M, Hanafusa H, et al: Single nucleotide polymorphism of the AXIN2 gene is preferentially associated with human lung cancer risk in a Japanese population. Int J Mol Med 18: 279-284, 2006.

11. Jang JS, Kim KM, Choi JE, et al: Identification of polymorphisms in the Caspase-3 gene and their association with lung cancer risk. Mol Carcinog 47: 383-390, 2007.
12. Zhai R, Liu G, Zhou W, et al: Vascular endothelial growth factor genotypes, haplotypes, gender, and the risk of non-small cell lung cancer. Clin Cancer Res 14: 612-617, 2008.

13. Chen Y, Sun R, Han W, Zhang Y, Song Q, Di C and Ma D: Nuclear translocation of PDCD5 (TFAR19): an early signal for apoptosis? FEBS Lett 509: 191-196, 2001.

14. Chen LN, Wang Y, Ma DL and Chen YY: Short interfering RNA against the PDCD5 attenuates cell apoptosis and caspase-3 activity induced by Bax overexpression. Apoptosis 11: 101-111, 2006.

15. Spinola M, Meyer $\mathrm{P}$, Kammerer $\mathrm{S}$, et al: Association of the PDCD5 locus with lung cancer risk and prognosis in smokers. J Clin Oncol 24: 1672-1678, 2006.

16. Ju W, Kim JW, Park NH, Song YS, Kim SC, Kang SB and Lee HP: Matrix metalloproteinase-1 promoter polymorphism and epithelial ovarian cancer: does ethnicity matter? J Obstet Gynaecol Res 33: 155-160, 2007.

17. Nurfadhlina M, Foong K, Teh LK, Tan SC, Mohd Zaki S and Ismail R: CYP2A6 polymorphisms in Malays, Chinese and Indians. Xenobiotica 36: 684-692, 2006. 